\title{
Bis(pentamethylcyclopentadienyl)keton und -thioketon: Kohlenstoff-Verbindungen mit präformierter Diels-Alder-Geometrie
}

\author{
Peter Jutzi*, Karl-Heinz Schwartzen, Andreas Mix, Hans-Georg Stammler und Beate Neumann \\ Fakultät für Chemie der Universität Bielefeld, \\ Universitätsstraße 25, W-4800 Bielefeld
}

Eingegangen am 23. Juni 1992

Key Words: 1,3-Cyclopentadiene-5-carbonyl chloride, 1,2,3,4,5-pentamethyl- / 1,3-Cyclopentadiene-5-carbothioyl chloride, 1,2,3,4,5-pentamethyl- / Ketone, bis(1,2,3,4,5-pentamethyl-1,3-cyclopentadien-5-yl) / Thioketone, bis(1,2,3,4,5pentamethyl-1,3-cyclopentadien-5-yl) / Diels-Alder preformation

\begin{abstract}
Bis(pentamethylcyclopentadienyl) Ketone and Thioketone: Carbon Compounds with Performed Diels-Alder Geometry
1,2,3,4,5-Pentamethyl-1,3-cyclopentadien-5-carbonyl chloride (2) is formed in good yields by the reaction of pentamethylcyclopentadienyllithium (1) with phosgene. The corresponding carbothioyl chloride 3 is synthesized by treatment of 1 with thiophosgene. Both acyl chlorides are stable against air and moisture and difficult to attack in $\mathrm{S}_{\mathrm{N}}$ 2-type reactions. Treatment of $\mathbf{2}$ and $\mathbf{3}$ with trimethyl(pentamethylcyclopenta-

leads to bis(1,2,3,4,5-pentamethyl-1,3-cyclopentadien-5-yl) ketone (5) and thioketone (6), respectively. Even at room temperature, 5 and $\mathbf{6}$ tend to intramolecular $[4+2]$ cycloaddition reactions. X-ray crystal structure investigations of 2,5 , and 6 show the steric demand of the pentamethylcyclopentadienyl ligand and explain the untypical chemical behavior of 2 and the easy $[2+4]$ cycloaddition reactions of 5 and $\mathbf{6}$.
\end{abstract} dienyl)stannane in the presence of boron trifluoride-ether
Verbindungen mit einem Pentamethylcylopentadienyl$\left(\mathrm{Cp}^{*}\right)$-Liganden an einem $\mathrm{sp}^{2}$-hybridisierten KohlenstoffAtom sind bereits bekannt. Neben Pentamethylcyclopentadiencarbonsäure-ethylester ${ }^{[1]}$ und Pentamethylcyclopentadienylcarbaldehyd ${ }^{[2]}$ sind auch einige $\mathrm{Cp}^{*}$-substituierte Ketone beschrieben ${ }^{[3-6]}$. Spektroskopische Untersuchungen zeigen, daß sich diese Ketone in Gegenwart von Aluminiumtrichlorid bereits bei tiefen Temperaturen hoch dynamisch verhalten ${ }^{[4]}$.

Bereits vor einigen Jahren haben wir unsere Untersuchungen an Pentamethylcyclopentadienyl-Verbindungen der schwereren Elemente aus der 14. Gruppe auch auf das Element Kohlenstoff ausgedehnt ${ }^{[7]}$. Im Hintergrund dieser Forschungsarbeiten steht die Idee, auch die zweiwertige Stufe des Kohlenstoffs durch $\pi$-Koordination von zwei Pentamethylcyclopentadienyl-Liganden zu stabilisieren. Für die schweren Homologen ${ }^{[8-10]}$ ist dies bereits realisiert. In diesem Zusammenhang sind für uns Kohlenstoff-Verbindungen mit einem und insbesondere mit zwei Pentamethylcyclopentadienyl-Substituenten als potentielle Vorstufen von besonderem Interesse. Aufbauend auf unsere Untersuchungen zum Reaktionsverhalten von Pentamethylcyclopentadienyllithium mit Halogenmethanen und Formylverbindungen ${ }^{[7]}$ berichten wir nachfolgend über Synthesen von bisher unbekannten mono- und bis-Cp${ }^{*}$-substituierten $\mathrm{C}_{1}$-Verbindungen mit $\mathrm{sp}^{2}$-hybridisiertem Zentralatom, beschreiben deren chemisches Verhalten und diskutieren strukturelle Besonderheiten dieser Verbindungen im Festkörper.

\section{Ergebnisse}

Wir haben früher bereits das Pentamethylcyclopentadiencarbonylchlorid 2 durch Einleiten von Kohlendioxid in eine
Suspension von Pentamethylcyclopentadienyllithium (1) in THF und Umsetzung des resulierenden Carboxylats mit Thionylchlorid erhalten ${ }^{[7]}$. Die Ausbeuten sind nach diesem Verfahren aber relativ gering. Einen wesentlich eleganteren und effektiveren Zugang zu dieser Verbindung bietet die Umsetzung von 1 mit Phosgen: Tropft man eine Lösung von Phosgen in Toluol bei $-30^{\circ} \mathrm{C}$ langsam zu einer Suspension von 1 in Hexan, so färbt sich die Reaktionsmischung sofort blaßgelb. Nach Abtrennen des Lithiumchlorids und Einengen des Filtrats kristallisiert das Säurechlorid 2 bei $-80^{\circ} \mathrm{C}$ (Ausbeute $80 \%$, Schema 1).

Verbindung 2 zeigt ein für Säurechloride äußerst ungewöhnliches chemisches Verhalten. Eine Substituion des Halogen-Atoms im Sinne einer $\mathrm{S}_{\mathrm{N}}$ 2-Reaktion scheint generell nicht möglich. So ist dieses Säurechlorid beispielsweise gegenüber Wasser stabil. Auch nach Erhitzen mit einer wäßrigen konzentrierten Ammoniak-Lösung in THF kann 2 quantitaiv zurückgewonnen werden. Mit Natriumamid in Toluol reagiert 2 selbst in der Siedehitze nicht.

\section{Schema 1}

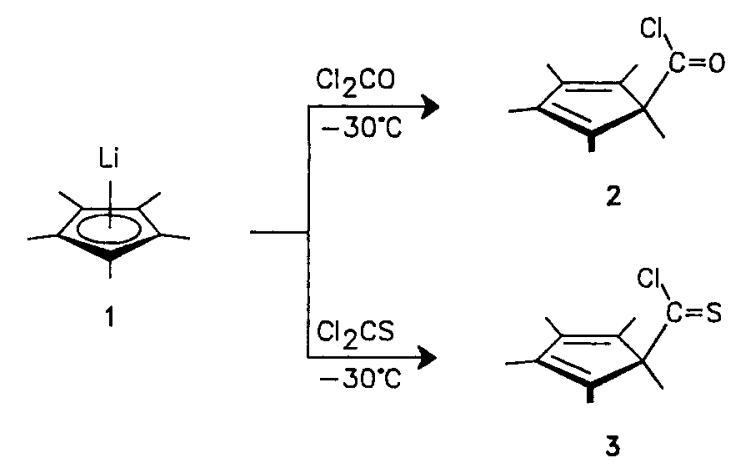


Analog zur Synthese von 2 ist auch das entsprechende Carbothioylchlorid 3 zugänglich. Versetzt man eine Suspension von 1 in Hexan mit einer Lösung von Thiophosgen in Hexan bei $-30^{\circ} \mathrm{C}$, so erhält man nach Abtrennen des Lithiumchlorids und Einengen des Filtrats 1,2,3,4,5-Pentamethyl-1,3-cyclopentadien-5-carbothioylchlorid (3) als dunkelrotes OOl in 80 proz. Ausbeute. Bei $-30^{\circ} \mathrm{C}$ kristallisiert die Verbindung in roten Nadeln. Sie besitzt einen intensiven Geruch und ist beständig gegenüber Luft und Feuchtigkeit. Die Neigung zur Polymerisation ist im Gegensatz zu anderen aliphaischen Carbothioylchloriden äußerst gering. So läßt sich 3 beispielsweise durch Vakuumdestillation reinigen. Unter Normalbedingungen beobachtet man erst nach einigen Tagen Polymerenbildung unter Schwarzfärbung.

Die ungewöhnliche Reaktionsträgheit der Säurechloride $\mathbf{2}$ und $\mathbf{3}$ dokumentiert, wie effektiv das jeweilige zentrale $\mathrm{sp}^{2}$ C-Atom bereits durch einen PentamethylcyclopentadienylSubstituenten abgeschirmt wird.

\section{Festkörperstruktur von $2^{[16,17]}$}

Um Informationen über den sterischen Anspruch des Cp*-Systems in den Säurechloriden zu erhalten, haben wir von Verbindungen 2 exemplarisch eine Röntgenstrukturanalyse angefertigt (Tab. 1 und 2, Abb. 1).

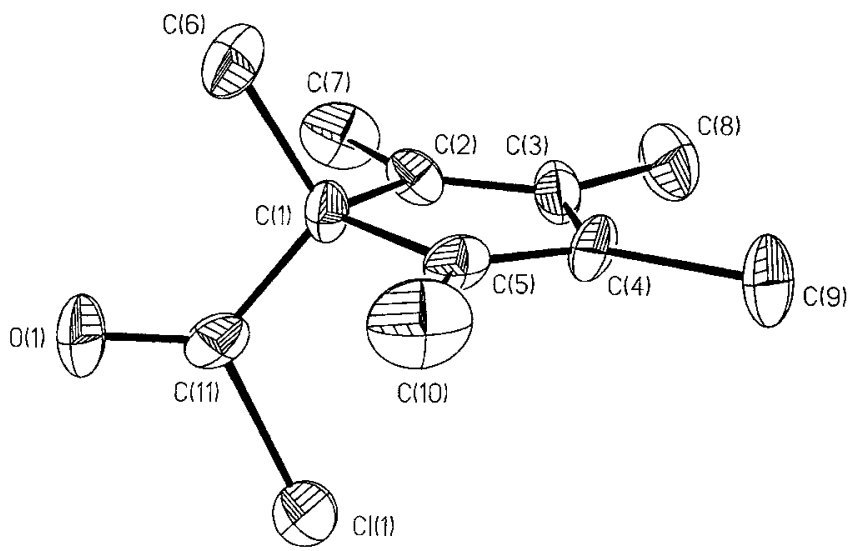

Abb. 1. Struktur von 2 im Festkörper; Bindungslängen $[\AA]$ : $\mathrm{C}(1)-\mathrm{C}(2) \quad 1.510(9), \quad \mathrm{C}(2)-\mathrm{C}(3) 1.326(9), \quad \mathrm{C}(3)-\mathrm{C}(4) 1.443(11)$, $\mathrm{C}(1)-\mathrm{C}(11)$ 1.781(7), $\mathrm{O}(1)-\mathrm{C}(11) 1.168(8), \mathrm{Cl}(1)-\mathrm{C}(11)$ 1.781(7); Bindungswinkel $\left[{ }^{\circ}\right]$ : $\mathrm{O}(1)-\mathrm{C}(11)-\mathrm{C}(1)$ 127.8(6), $\mathrm{Cl}(1)-\mathrm{C}(11)-$

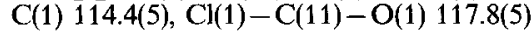

Das Säurechlorid liegt im Festkörper in diskreten, monomeren Einheiten vor. Die Elementarzelle enthält vier Moleküle. Die Bindungslängen und -winkel zeigen, daß der Pentamethylcyclopentadienyl-Substituent monohapto an die COCl-Einheit gebunden ist. Das Ringsystem ist planar. Die Bindungslängen liegen in den jeweils erwarteten Bereichen. Der enorme sterische Anspruch des Cp*-Liganden wirkt sich auf die räumliche Orientierung der $\mathrm{COCl}$-Einheit aus. Diese ist so ausgerichtet, daß das Chlor-Atom unterhalb der Ringebene steht. Obwohl die COCl-Einheit auf diese Art offensichtlich die räumlich günstigste Anordnung einnimmt, wird die ideale Geometrie des Carbonyl-Kohlenstoffatoms (120-Winkel) durch den Raumanspruch des Carbonyl-Sau- erstoffatoms und der allyständigen Methylgruppe an $\mathrm{C}(1)$ merklich deformiert. So ist der Winkel $\mathrm{C}(1)-\mathrm{C}(11)-\mathrm{O}(1)$ mit $127.8^{\circ}$ deutlich aufgeweitet, während gleichzeitig der Winkel $\mathrm{C}(1)-\mathrm{C}(11)-\mathrm{Cl}(1)$ auf $114.4^{\circ}$ reduziert ist.

Tab. 1. Kristallstrukturdaten von 2, 5 und $\mathbf{6}$

\begin{tabular}{|c|c|c|c|}
\hline & 2 & 5 & 6 \\
\hline Summenformel & $\mathrm{C}_{11} \mathrm{H}_{15} \mathrm{ClO}$ & $\mathrm{C}_{21} \mathrm{H}_{30} \mathrm{O}$ & $\mathrm{C}_{21} \mathrm{H}_{30} \mathrm{~S}$ \\
\hline Molmasse & 198.7 & 298.5 & 314.5 \\
\hline \multirow[t]{6}{*}{ Zelldaten $(\AA)$} & $\mathrm{a}=7.633(3)$ & $a=8.331(3)$ & $a=7.988(5)$ \\
\hline & $\mathrm{b}=11.531(5)$ & $b=8.710(5)$ & $\mathrm{b}=15.156(10)$ \\
\hline & \multirow[t]{4}{*}{$c=12.380(5)$} & $c=14.613(4)$ & \multirow[t]{2}{*}{$\mathrm{c}=15.339(9)$} \\
\hline & & $\alpha=83.76(4)$ & \\
\hline & & $\beta=79.64(3)$ & \multirow[t]{2}{*}{$\beta=93.10(5)$} \\
\hline & & $\gamma=61.97(3)$ & \\
\hline Zellvolumen $\left(\AA^{3}\right)$ & $1089.8(8)$ & $920.4(6)$ & $1857(4)$ \\
\hline Kristallsystem & orthorhombisch & triklin & monoklin \\
\hline Raumgruppe & $\mathrm{P} 21_{1} 2_{1}$ & $\mathrm{P} \overline{1}$ & $\mathrm{C} 2 / \mathrm{c}$ \\
\hline $\mathrm{d}_{\mathrm{c}}$ & $1.211 \mathrm{~g} / \mathrm{cm}^{3}$ & $1.077 \mathrm{~g} / \mathrm{cm}^{3}$ & $1.125 \mathrm{~g} / \mathrm{cm}^{3}$ \\
\hline$Z, F(000)$ & 4,424 & 2,328 & 4,688 \\
\hline \multirow[t]{2}{*}{ Strahlung } & \multicolumn{3}{|c|}{ Mo-K $\alpha, \lambda=0.71073 \AA$} \\
\hline & $\mu=0.309 \mathrm{~mm}^{-1}$ & $\mu=0.059 \mathrm{~mm}^{-1}$ & $\mu=0.163 \mathrm{~mm}^{-1}$ \\
\hline $2 \theta \min / \max$ & $3.0 / 55.0$ & $3.0 / 50.0$ & $3.0 / 55.0$ \\
\hline Meßtemperatur & $183 \mathrm{~K}$ & $190 \mathrm{~K}$ & $193 \mathrm{~K}$ \\
\hline Gemessene Reflexe & 1473 & 3312 & 2533 \\
\hline Beobachte Reflexe & $902(\mathrm{~F}>3.0 \sigma(\mathrm{F}))$ & 1991 & 1228 \\
\hline Absorptionskorrektur & XABS[16] & N/A & $\mathrm{N} / \mathrm{A}$ \\
\hline \multicolumn{4}{|l|}{ Zahl der verfeinerten } \\
\hline Parameter & 118 & 199 & 101 \\
\hline $\mathrm{R}$ & 0.075 & 0.093 & 0.058 \\
\hline $\mathrm{R}_{\mathrm{W}}$ & 0.050 & 0.102 & 0.053 \\
\hline
\end{tabular}

Die Kristallstruktur von 2 zeigt, daß sterische Effekte für das ungewöhnlich inerte Reaktionsverhalten des Säurechlorids 2 verantwortlich sind. Der $\mathrm{Cp}^{*}$-Substituent schirmt die Carbonyl-Einheit nahezu perfekt ab. Ein nucleophiler Angriff an das Kohlenstoff-Zentrum der COCl-Funktion wird so wesentlich erschwert.

\section{Bis(pentamethylcyclopentadienyl)keton und -thioketon}

Trotz der äußerst effektiven Abschirmung der Chlorcarbonyl- bzw. Chlorthiocarbonyl-Einheit in den Säurechloriden 2 und 3 ist eine Substiution des Halogen-Atoms möglich. Beide Säurechloride werden in Gegenwart von Halogenid-Akzeptoren so aktiviert, daß eine Substitution des Halogen-Atoms im Sinne einer $\mathrm{S}_{\mathrm{N}} 1$-Reaktion möglich wird. Wir konnten zeigen, daß dabei ensprechende Acyl- bzw. Thioacyl-Kationen als reaktive Zwischenstufen auftreten ${ }^{[11]}$.

Die Säurechloride 2 und 3 sollten sich dementsprechend auch als Vorstufen zur Darstellung von Bis(pentamethylcyclopentadienyl)-keton (5) bzw. der entsprechenden ThioVerbindung 6 eignen. Setzt man 2 mit Trimethyl(pentamethylcyclopentadienyl)stannan (4) in Gegenwart katalytischer Mengen Bortrifluorid - Diethylether um, so tritt allmählich eine Farbvertiefung der Reaktionsmischung von gelb nach gelbbraun ein. Nach einer Reaktionszeit von 12 Stunden bei 
Raumtemperatur und anschließender alkalisch-wäßriger Aufarbeitung isoliert man 5 in 40proz. Ausbeute (Schema 2). Aus Hexan kristallisiert die Verbindung bei $-30^{\circ} \mathrm{C}$ in Form dünner, farbloser Blättchen. Unter vergleichbaren Bedingungen reagiert auch das Carbothioylchlorid 3. Tropft man $\mathrm{Et}_{2} \mathrm{O} \cdot \mathrm{BF}_{3}$ bei $0^{\circ} \mathrm{C}$ langsam zu einer Hexan-Lösung der Thio-Verbindung 3 und des Stannans 4 , so färbt sich die Reaktionsmischung rotbraun. Nach 12stündigem Rühren, wobei die Mischung allmählich auf Raumtemperatur erwärmt wird, und entsprechender Aufarbeitung wird Bis(1,2,3,4,5-pentamethyl-1,3-cyclopentadien-5-yl)thioketon (6) in 40proz. Ausbeute isoliert (Schema 2).

Schema 2

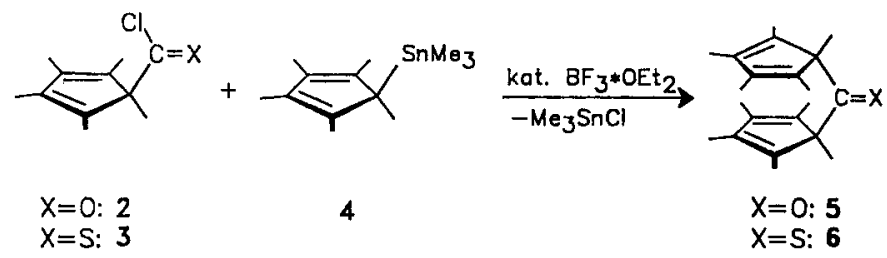

Die Ketone 5 und 6 sind die ersten Verbindungen, in denen ein Kohlenstoff-Atom zwei Pentamethylcyclopentadienyl-Substituenten trägt.

\section{Chemisches Verhalten der Ketone 5 und 6}

Die Schwefel-Verbindung 6 zeigt einmal mehr das hohe Potential des $\mathrm{Cp}^{*}$-Liganden, labile Struktureinheiten kinetisch effektiv zu stabilisieren. Während sich Thioketone mit kleinen Alkylsubstituenten bereits bei niedrigen Temperaturen rasch unter Polymerenbildung zersetzen, zeigt die CSEinheit in 6 unter Normalbedingungen keine Polymerisationstendenz und ist darüber hinaus luft- und hydrolysestabil. Dagegen tendieren die $\mathrm{Cp}^{*}$-Liganden bereits bei Raumtemperatur zur intramolekularen Diels-Alder-Reaktion. Diese Cycloaddition ist offensichtlich eine Folge der räumlichen Nähe der beteiligten $\pi$-Systeme ${ }^{[12]}$. Als Produkt dieser $[4+2]$-Cycloaddition entsteht die tetracyclische Thio-Verbindung 8 (Schema 3). Die Reaktion läuft sowohl in Lösung als auch im Festkörper ab. Im Verlauf der Cyclisierung wird eine Farbänderung von rot nach rosa beobachtet. In Lösung sind vom Thioketon 6 unter Normalbedingungen nach 24 Stunden bereits $50 \%$ zum Polycyclus 8 cyclisiert. Die intramolekulare Diels-Alder-Reaktion läßt sich bei 6 auch photochemisch induzieren. Die Reaktionsgeschwindigkeit ist hierbei extrem hoch. So cyclisiert 6 in Hexan unter Bestrahlung selbst bei tiefen Temperaturen innerhalb von Sekunden zum Thioketon 8. Im Vergleich zur Schwefel-Verbindung ist die Diels-Alder-Neigung des Ketons 5 erheblich geringer. Lagert man die Verbindung bei Raumtemperatur, so haben sich nach einer Woche erst 5\% in den Tetracyclus 7 umgewandelt (Schema 3).

Die tetracyclischen Systeme 7 und 8 lassen sich auch gezielt synthetisieren. Erhitzt man das Keton 5 einige Stunden in Benzol unter Rückfluß, so erhält man nach Entfernen des Solvens Verbindung 7 als farbloses Pulver in quantitiativer Ausbeute. Auf analoge Weise läßt sich aus 6 quantitativ die entsprechende Thio-Verbindung 8 als rosarotes Pulver darstellen.

Schema 3
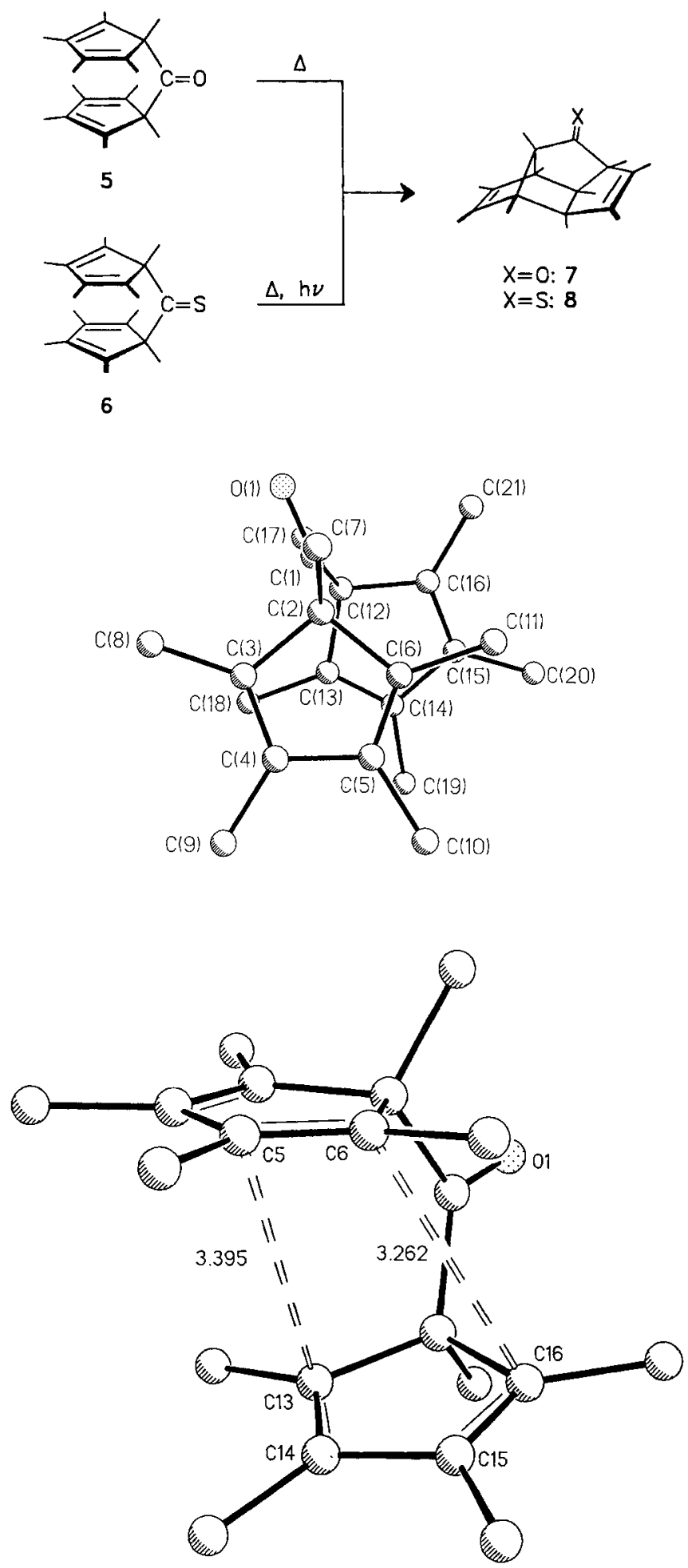

Abb. 2. Festkörperstruktur von 5 (oben: Aufsicht, unten: Blick in Richtung $\mathrm{C}-\mathrm{O}$-Bindung); Bindungslängen $[\AA]: \quad \mathrm{O}(1)-\mathrm{C}(1)$ $1.208(8), \quad C(1)-C(2) \quad 1.562(8), \quad C(2)-C(3) \quad 1.532(8), \quad C(3)-C(4)$ 1.338(9), $C(4)-C(5)$ 1.462(12); Bindungswinkel [ $]$ : $O(1)-C(1)-$ $\mathrm{C}(2) 118.5(5), \mathrm{C}(2)-\mathrm{C}(1)-\mathrm{C}(12) 121.9(5), \mathrm{C}(1)-\mathrm{C}(2)-\mathrm{C}(3)$ 108.1(5), $\mathrm{C}(1)-\mathrm{C}(2)-\mathrm{C}(6) 117.1(5)$ 


\section{Festkörperstrukturen von 5 und $6^{[17]}$}

Um Informationen über die räumliche Anordnung der Pentamethylcyclopentadienyl-Liganden und die von diesen Liganden ausgehenden sterischen Zwänge in den Ketonen 5 und $6 \mathrm{zu}$ erhalten, haben wir von beiden Verbindungen Röntgenstrukturanalysen durchgeführt. (Tab. 1, 3 und 4).
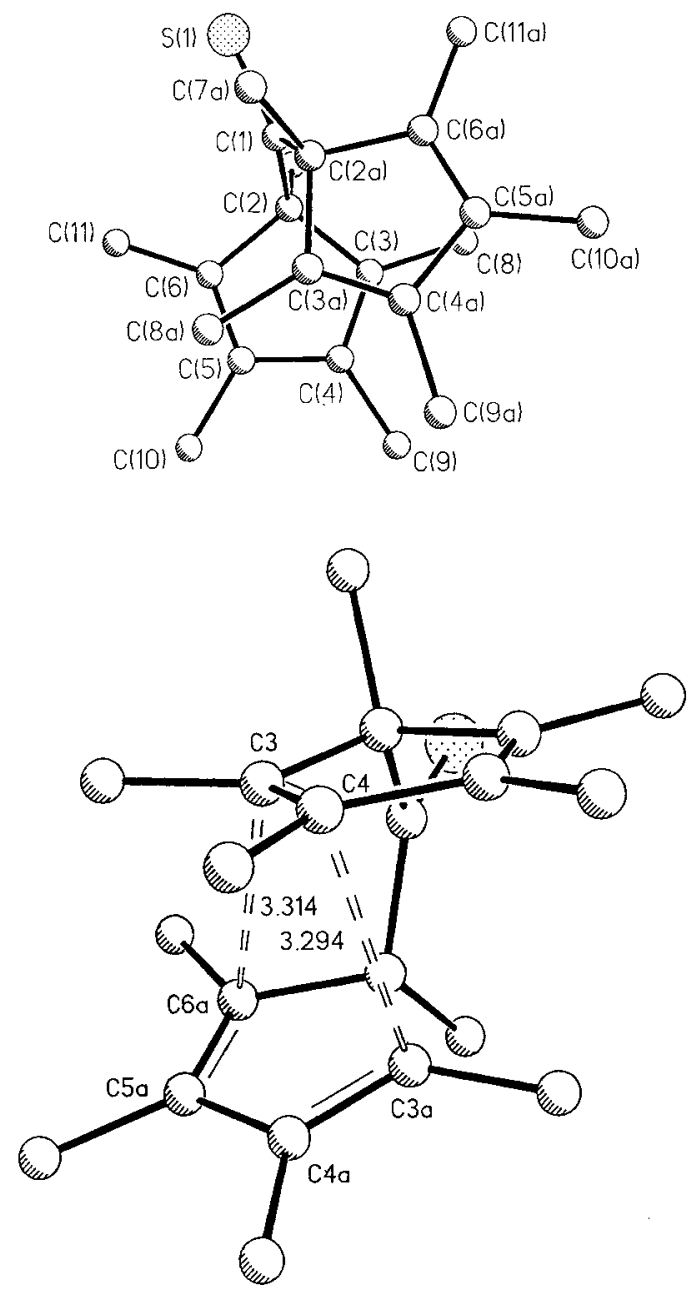

Abb. 3. Festkörperstruktur von 6 (oben: Aufsicht, unten: Blick in Richtung C-S-Bindung); Bindungslängen $[\AA]$ : $S(1)-C(1) 1.631(5)$ $\mathrm{C}(1)-\mathrm{C}(2)$ 1.530(4), $\mathrm{C}(2)-\mathrm{C}(3)$ 1.526(6), $\mathrm{C}(3)-\mathrm{C}(4) 1.330(5)$, $C(4)-C(5)$ 1.453(5); Bindungswinkel $\left[{ }^{\circ}\right]: S(1)-C(1)-C(2)$ 119.1(5), $\mathrm{C}(2)-\mathrm{C}(1)-\mathrm{C}(2 \mathrm{a}) 121.8(4), \mathrm{C}(1)-\mathrm{C}(2)-\mathrm{C}(3)$ 117.3(3), $\mathrm{C}(1)-\mathrm{C}(2)-$ C(6) $108.5(2)$

Die Abb. 2 und 3 zeigen verschiedene Ansichten der Strukturen von 5 und 6. Die Verbindungen liegen im Festkörper als diskrete monomere Einheiten vor. Die Elementarzellen enthalten 2 bzw. 4 Moleküle der erwarteten $\mathrm{Zu}$ sammensetzung. Beide Verbindungen sind strukturell ähnlich aufgebaut. Die Bindungslängen liegen in den erwarteten Bereichen. Die Ringsysteme sind in beiden Fällen planar. Dagegen ist die Anordnung der $\mathrm{Me}_{5} \mathrm{C}_{5}$-Einheiten zueinander nicht coplanar. Die Ebenen-Normalen schließen in beiden Derivaten einen Winkel von ca. $162^{\circ}$ ein. Der Bindungswinkel am zentralen Kohlenstoff-Atom ist sowohl in 5 als auch in 6 mit $121.8^{\circ}$ gegenüber dem Idealwert für ein $\mathrm{sp}^{2}$ Hybrid geringfügig aufgeweitet.

Beide Strukturen werden maßgeblich durch den enormen räumlichen Anspruch der beiden $\mathrm{Cp}^{*}$-Substituenten dominiert. So macht die sterische Wechselwirkung zwischen den Heteroatomen an den zentralen $\mathrm{C}_{1}$-Einheiten und den allylständigen Methylgruppen eine Verdrehung der $\mathrm{Cp}^{*}$-Liganden gegeneinander notwendig. Es resultiert eine stufenförmige Anordnung der Ringsysteme. Durch die Torsion der Cp*-Liganden sind die Methylgruppen an den Allyl-Positionen in beiden Verbindungen nicht direkt über oder unter den Heteroatomen, sondern seitlich versetzt angeordnet. Im Thioketon beträgt der Torsionswinkel $46.8^{\circ}$. Die Verdrehung der $\mathrm{Cp}^{*}$-Liganden führt in 6 zu einer ungünstigen Staffelung der Methylgruppen $\mathrm{C}(9) / \mathrm{C}(9 \mathrm{a})$ und $\mathrm{C}(10) / \mathrm{C}(8 \mathrm{a})$. Durch den geringeren Raumbedarf des Sauerstoff-Atoms im Keton 5 ist hier ein Torsionswinkel von $39.5^{\circ}$ zur Minimierung der sterischen Wechselwirkung ausreichend.

Anhand der Festkörperstrukturen läßt sich auch die Tendenz beider Verbindungen zur intramolekularen Diels-Alder-Reaktion zwanglos erklären. Betrachtet man den Abstand der Ringmittelpunkte zueinander, so ist im Thioketon 6 die kürzeste Distanz zweier $\mathrm{Me}_{5} \mathrm{C}_{5}$-Einheiten in der Reihe der bisher kristallographisch untersuchten Hauptgruppenelement-Verbindungen mit zwei $\mathrm{Cp}^{*}$-Liganden realisiert ${ }^{[13]}$. Setzt man den hier gefundenen Abstand von $3.38 \AA$ in $\mathrm{Re}$ lation zum van-der-Waals-Radius eines $\mathrm{Cp}^{*}$-Fragmentes (ca. $1.6 \AA)^{[14]}$, so ist nahezu ein Grenzwert in bezug auf die mögliche räumliche Annäherung zweier $\mathrm{Cp}^{*}$-Einheiten erreicht.

Im Thioketon 6 ist die Doppelbindung zwischen $\mathrm{C}(3)$ und C(4) durch die Torsion der beiden Ringsysteme nahezu rechtwinkelig oberhalb der Dien-Einheit, begrenzt durch $\mathrm{C}(3 \mathrm{a})$ und C(6a), angeordnet. Dies ist deutlich der Abb. $3 \mathrm{zu}$ entnehmen. Durch diese Anordnung von Dien und Dienophil und durch die mit 3.31 bzw. $3.29 \AA$ recht kurzen Abstände der direkt am Ringschluß beteiligten KohlenstoffAtome $\mathrm{C}(3)$ und $\mathrm{C}(6 \mathrm{a})$ sowie $\mathrm{C}(4)$ und $\mathrm{C}(3 \mathrm{a})$ ist in 6 eine Produkt-nahe Grundzustandsgeometrie realisiert, wodurch die intramolekulare Diels-Alder-Reaktion entropisch begünstigt wird. ${ }^{[15]}$.

Bedingt durch den geringeren Torsionswinkel der beiden Cp*-Einheiten im Keton $\mathbf{5}$ werden Dien und Dienophil hier nicht so exakt übereinander angeordnet wie in 6 (Abb. 2). Hieraus resultieren auch vergleichsweise größere Abstände zwischen den an der Cycloaddition beteiligten KohlenstoffAtomen $\mathrm{C}(5)$ und $\mathrm{C}(13)(3.39 \AA)$ sowie $\mathrm{C}(6)$ und $\mathrm{C}(16)$ $(3.26 \AA)$. Dementsprechend sind die geometrischen Voraussetzungen für eine intramolekulare Cycloaddition im Keton 5 nicht so günstig wie in der Schwefel-Verbindung 6. Dies bestätigt sich auch im Experiment.

Alle hier vorgestellten mono- und bis- $\mathrm{Cp}^{*}$-substituierten $\mathrm{C}_{1}$-Verbindungen dokumentieren eindrucksvoll die weitreichenden Konsequenzen, die sich aus dem sterischen Anspruch von $\sigma$-gebundenen PentamethylcyclopentadienylEinheiten ergeben können. So ist sowohl das ungewöhnlich inerte Verhalten der Säurechloride 2 und 3 als auch die geometrische Präformation eines Diels-Alder-Übergangs- 
zustandes im Thioketon 6 und weniger ausgeprägt auch im Keton 5 ausschließlich eine Folge der durch die Cp*-Liganden hervorgerufenen sterischen Zwänge.

\section{Experimenteller Teil}

Alle Arbeiten wurden unter Ausschluß von Luft und Feuchtigkeit unter Argon durchgeführt; verwendete Geräte, Chemikalien und Lösungsmittel waren entsprechend vorbereitet. - Schmelzpunkte: Büchi-510-Schmelzpunktbestimmungsapparatur, Probenröhrchen abgeschmolzen. - NMR (Bruker AM 300): ${ }^{1} \mathrm{H}$ (300.1 MHz) ext. TMS, ${ }^{13} \mathrm{C}\left\{{ }^{1} \mathrm{H}\right\}(75.4 \mathrm{MHz})$ ext. TMS. - MS: Varian CH $5(70 \mathrm{eV}$, $300 \mu \mathrm{A}$ Emission); es sind nur charakteristische Fragment-Ionen angegeben. - CHN-Analysen: Mikroanalytisches Laboratorium Beller, Göttingen; Analytisches Labor der Fakultät für Chemie der Universität Bielefeld.

1,2,3,4,5-Pentamethyl-1,3-cyclopentadien-5-carbonylchlorid (2): $\mathrm{Zu}$ einer Lösung von $13.6 \mathrm{~g}(0.10 \mathrm{~mol})$ Pentamethylcyclopentadien in $300 \mathrm{ml}$ Hexan werden bei Raumtemp. $62.5 \mathrm{ml}$ einer $1.60 \mathrm{M}$ Lösung von $n$-Butyllithium in Hexan $(0.10 \mathrm{~mol})$ getropft. Man rührt $12 \mathrm{~h}$, kühlt auf $-30^{\circ} \mathrm{C}$ ab und tropft langsam $51.8 \mathrm{ml}$ einer $1.93 \mathrm{M} \mathrm{Lö-}$ sung von Phosgen in Toluol $(0.10 \mathrm{~mol}) \mathrm{zu}$. Anschließend wird die Reaktionsmischung auf Raumtemp. erwärmt und noch $4 \mathrm{~h}$ gerührt. Man filtriert vom Lithiumchlorid ab und engt das blaßgelbe Filtrat i. Vak. ein. Kristallisation bei $-80^{\circ} \mathrm{C}$ liefert 2 in farblosen Nadeln. Ausb. $15.8 \mathrm{~g}(80 \%)$, Schmp. $41^{\circ} \mathrm{C} .-{ }^{1} \mathrm{H}-\mathrm{NMR}\left(\mathrm{C}_{6} \mathrm{D}_{6}\right): \delta=1.13$ (s, $\left.3 \mathrm{H}, \mathrm{CH}_{3}\right), 1.53\left(\mathrm{~s}, 6 \mathrm{H}, \mathrm{CH}_{3}\right), 1.63\left(\mathrm{~s}, 6 \mathrm{H}, \mathrm{CH}_{3}\right) .-{ }^{13} \mathrm{C}-\mathrm{NMR}\left(\mathrm{C}_{6} \mathrm{D}_{6}\right)$ : $\delta=10.02\left(\mathrm{CH}_{3}\right), 11.29\left(\mathrm{CH}_{3}\right), 17.26\left(\mathrm{CH}_{3}\right), 72.48\left(\mathrm{C}-\mathrm{CH}_{3}\right), 136.28$ $\left(\mathrm{C}-\mathrm{CH}_{3}\right), 140.55\left(\mathrm{C}-\mathrm{CH}_{3}\right), 176.60(\mathrm{COCl}) .-\mathrm{MS}, \mathrm{m} / z(\%):\left[\mathrm{M}^{+}\right]$ $198(22),\left[\mathrm{M}^{+}-\mathrm{CO}\right] 170(63),\left[\mathrm{M}^{+}-\mathrm{Cl}\right] 163(5),\left[\mathrm{M}^{+}-\mathrm{CO}\right.$ - Me] $155(8),\left[\mathrm{Me}_{5} \mathrm{C}_{5}^{+}\right] 135(100),\left[\mathrm{Me}_{5} \mathrm{C}_{5}^{+}\right] 134$ (17).

\section{$\mathrm{C}_{11} \mathrm{H}_{15} \mathrm{ClO}(198.7)$ Ber. C 66.50 H 7.61 Gef. C 66.32 H 7.68}

1,2,3,4,5-Pentamethyl-1,3-cyclopentadien-5-carbothioylchlorid (3): $\mathrm{Zu}$ einer Lösung von $13.6 \mathrm{~g}(0.10 \mathrm{~mol})$ Pentamethylcyclopentadien in $300 \mathrm{ml}$ Hexan werden bei Raumtemp. $62.5 \mathrm{ml}$ einer $1.60 \mathrm{M}$ Lösung von $n$-Butyllithium in Hexan $(0.10 \mathrm{~mol})$ getropft. Man rührt $12 \mathrm{~h}$, kühlt auf $-30^{\circ} \mathrm{C}$ ab und tropft langsam $11.5 \mathrm{~g}(0.10 \mathrm{~mol})$ Thiophosgen, gelöst in $20 \mathrm{ml}$ Hexan, zu. Anschließend wird die Reaktionsmischung auf Raumtemp. erwärmt und noch $4 \mathrm{~h}$ gerührt. Man filtriert vom Lithiumchlorid ab und engt das orangerote Filtrat i.Vak. ein. Nach fraktionierter Destillation i.Vak. erhält man 3 als rote, ölige Flüssigkeit. Ausb. $15.2 \mathrm{~g}(71 \%)$, Sdp. $62^{\circ} \mathrm{C} / 2 \mathrm{mbar}$. - ${ }^{1} \mathrm{H}-\mathrm{NMR}\left(\mathrm{C}_{6} \mathrm{D}_{6}\right): \delta=1.33\left(\mathrm{~s}, 3 \mathrm{H}, \mathrm{CH}_{3}\right) ; 1.60\left(\mathrm{~s}, 6 \mathrm{H}, \mathrm{CH}_{3}\right), 1.63$ (s, 6H, CH 3$) .-{ }^{13} \mathrm{C}-\mathrm{NMR}\left(\mathrm{C}_{6} \mathrm{D}_{6}\right): \delta=9.72\left(\mathrm{CH}_{3}\right), 10.29\left(\mathrm{CH}_{3}\right)$, $21.87\left(\mathrm{CH}_{3}\right), 78.44\left(\mathrm{C}-\mathrm{CH}_{3}\right), 138.18\left(\mathrm{C}-\mathrm{CH}_{3}\right), 139.63\left(\mathrm{C}-\mathrm{CH}_{3}\right)$, 220.10 (CSCl). - MS, $m / z(\%):\left[\mathrm{M}^{+}\right] 214(69),\left[\mathrm{M}^{+}-\mathrm{Me}\right] 199$ (15), $\left[\mathrm{M}^{+}-\mathrm{SH}\right], 181(60),\left[\mathrm{M}^{+}-\mathrm{Cl}\right] 179(100),\left[\mathrm{M}^{+}-\mathrm{Cl}-\right.$ $\mathrm{Me}], 164$ (30), [ $\left.\mathrm{Me}_{5} \mathrm{C}_{5}^{+}\right], 135$ (93).

\section{$\mathrm{C}_{11} \mathrm{H}_{15} \mathrm{ClS}$ (214.8) Ber. C 61.52 H 7.04 Gef. C 61.58 H 7.18}

Bis (1,2,3,4,5-pentamethyl-1,3-cyclopentadien-5-yl)keton (5): $11.9 \mathrm{~g}(60.0 \mathrm{mmol})$ des Säurechlorids 2 und $17.9 \mathrm{~g}$ Trimethyl(pentamethylcyclopentadienyl)stannan (4) werden in $120 \mathrm{ml} \mathrm{Hexan} \mathrm{ge-}$ löst und mit $3.3 \mathrm{ml}$ Bortrifluorid - Diethylether versetzt. Man rührt $12 \mathrm{~h}$. Danach wird die Reaktionsmischung mit $50 \mathrm{ml}$ einer wäßrigen Natriumcarbonat-Lösung versetzt. Die organische Phase wird abgetrennt, nochmals mit Wasser gewaschen und mit Magnesiumsulfat getrocknet. Einengen und Kristallisation bei $-80^{\circ} \mathrm{C}$ liefert 5

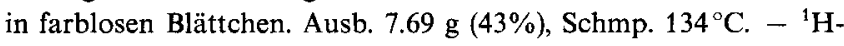
NMR $\left(\mathrm{C}_{6} \mathrm{D}_{6}\right): \delta=1.25\left(\mathrm{~s}, 6 \mathrm{H}, \mathrm{CH}_{3}\right), 1.55\left(\mathrm{~s}, 12 \mathrm{H}, \mathrm{CH}_{3}\right), 1.59(\mathrm{~s}$, $\left.12 \mathrm{H}, \mathrm{CH}_{3}\right),-{ }^{13} \mathrm{C}-\mathrm{NMR}\left(\mathrm{C}_{6} \mathrm{D}_{6}\right) ; \delta=11.25\left(\mathrm{CH}_{3}\right), 11.39\left(\mathrm{CH}_{3}\right), 19.93$ $\left(\mathrm{CH}_{3}\right), 71.40\left(\mathrm{C}-\mathrm{CH}_{3}\right), 136.01\left(\mathrm{C}-\mathrm{CH}_{3}\right), 138.37\left(\mathrm{C}-\mathrm{CH}_{3}\right), 207.66$
(CO). - MS, $m / z(\%):\left[\mathrm{M}^{+}\right] 298(13),\left[\mathrm{M}^{+}-\mathrm{Me}_{5} \mathrm{C}_{5}\right] 163(2)$, $\left[\mathrm{Me}_{5} \mathrm{C}_{5}^{+}\right] 135(100)$.

$\mathrm{C}_{21} \mathrm{H}_{30} \mathrm{O}$ (298.5) Ber. C 84.51 H 10.13 Gef. C 84.80 H 10.42

Bis(1,2,3,4,5-pentamethyl-1,3-cyclopentadien-5-yl)thioketon (6): $12.9 \mathrm{~g}(60.0 \mathrm{mmol}) 3$ und $17.9 \mathrm{~g} 4$ werden in $120 \mathrm{ml}$ Hexan gelöst und unter Eiskühlung mit $0.4 \mathrm{ml}$ Bortrifluorid-Diethylether versetzt. Man rührt $12 \mathrm{~h}$, wobei die Lösung langsam auf Raumtemp. erwärmt wird. Danach wird die Reaktionsmischung mit $50 \mathrm{ml}$ einer wäßrigen Natriumcarbonat-Lösung versetzt. Die organische Phase wird abgetrennt, nochmals mit Wasser gewaschen und mit Magnesiumsulfat getrocknet. Einengen und Kristallisation bei $-80^{\circ} \mathrm{C}$ liefert 6 in leuchtend roten Rhomben. Ausb. $8.77 \mathrm{~g} \mathrm{(46 \% ),} \mathrm{Schmp.}$

Tab. 2. Atomkoordinaten $\left(\cdot 10^{4}\right)$ und äquivalente isotrope Schwingungsparameter von 2 (mit Standardabweichungen)

\begin{tabular}{|c|c|c|c|c|c|c|c|c|}
\hline Atom & $x$ & & $y$ & & $z$ & & & $\overline{\mathrm{eq})}$ \\
\hline$c l(1)$ & 1463 & (2) & 5704 & (2) & 4603 & (1) & 42 & (1) \\
\hline$D(1)$ & 3744 & (6) & 7078 & (4) & 3821 & (4) & 51 & (2) \\
\hline$C(1)$ & 5022 & (8) & 5299 & (5) & 4449 & (5) & 22 & (2) \\
\hline$C(2)$ & 4732 & (8) & 4208 & (6) & 3799 & (5) & 25 & (2) \\
\hline$c(3)$ & 4604 & (8) & 3302 & (5) & 4453 & (7) & 30 & (3) \\
\hline$c(4)$ & 4700 & (9) & 3705 & (6) & 5581 & (6) & 29 & (3) \\
\hline$c(5)$ & 4934 & (8) & 4847 & (6) & 5608 & (5) & 26 & (2) \\
\hline$c(6)$ & 6816 & (7) & 5857 & (5) & 4208 & (4) & 33 & (2) \\
\hline$c(7)$ & 4652 & (8) & 4265 & (6) & 2566 & (4) & 43 & (3) \\
\hline$c(8)$ & 4430 & (9) & 2053 & (5) & 4143 & (5) & 47 & (3) \\
\hline$c(9)$ & 4592 & (10) & 2871 & (6) & 6517 & (5) & 52 & (3) \\
\hline$C(10)$ & 5131 & (10) & 5645 & (7) & 6544 & (5) & 49 & (3) \\
\hline$(11)$ & 3605 & (9) & 6170 & (6) & 4230 & (5) & 28 & (2) \\
\hline
\end{tabular}

Tab. 3. Atomkoordinaten $\left(\cdot 10^{4}\right)$ und äquivalente isotrope Schwingungsparameter von $\mathbf{5}$ (mit Standardabweichungen)

\begin{tabular}{|c|c|c|c|c|c|c|c|c|}
\hline Atom & $x$ & & $y$ & & $z$ & & & eq) \\
\hline$O(1)$ & -528 & (6) & 8310 & (6) & 2523 & (3) & 51 & (3) \\
\hline$C(1)$ & 1087 & (8) & 7859 & (7) & 2511 & (4) & 29 & (3) \\
\hline$c(2)$ & 1888 & (8) & 7281 & (7) & 3448 & (4) & 28 & (3) \\
\hline$c(3)$ & 2810 & (8) & 5290 & (7) & 3521 & (3) & 30 & (3) \\
\hline$C(4)$ & 4563 & (8) & 4717 & (8) & 3630 & (4) & 31 & (3) \\
\hline$C(5)$ & 4930 & (8) & 6194 & (8) & 3658 & (4) & 35 & (3) \\
\hline$C(6)$ & 3463 & (8) & 7665 & (8) & 3551 & (3) & 29 & (3) \\
\hline$C(7)$ & 277 & (9) & 8061 & (8) & 4251 & (4) & 42 & (3) \\
\hline$C(8)$ & 1758 & (10) & 4284 & (8) & 3495 & (4) & 47 & (4) \\
\hline$c(9)$ & 5977 & (9) & 2860 & (8) & 3733 & (5) & 55 & (3) \\
\hline$c(10)$ & 6790 & (9) & 5943 & (10) & 3796 & (5) & 59 & (4) \\
\hline$C(11)$ & 3150 & (11) & 9495 & (8) & 3644 & (4) & 53 & (4) \\
\hline$c(12)$ & 2319 & (8) & 7839 & (7) & 1564 & (4) & 27 & (3) \\
\hline$c(13)$ & 4261 & (8) & 6365 & (7) & 1417 & (3) & 25 & (2) \\
\hline$C(14)$ & 5450 & (8) & 7006 & (8) & 1276 & (3) & 28 & (3) \\
\hline$c(15)$ & 4447 & (8) & 8905 & (7) & 1342 & (4) & 28 & (3) \\
\hline$c(16)$ & 2615 & (8) & 9440 & (7) & 1495 & (3) & 29 & (3) \\
\hline$C(17)$ & 1242 & (9) & 7916 & (8) & 767 & (4) & 41 & (3) \\
\hline$C(18)$ & 4611 & (9) & 4512 & (7) & 1327 & (4) & 41 & (3) \\
\hline$c(19)$ & 7523 & (9) & 6010 & (9) & 1066 & (4) & 50 & (4) \\
\hline$C(20)$ & 5456 & (10) & 9982 & (8) & 1212 & (4) & 49 & (4) \\
\hline$C(21)$ & 1102 & (9) & 11238 & (7) & 1566 & (4) & 46 & (3) \\
\hline
\end{tabular}


$160^{\circ} \mathrm{C} .-{ }^{1} \mathrm{H}-\mathrm{NMR}\left(\mathrm{C}_{6} \mathrm{D}_{6}\right): \delta=1.45\left(\mathrm{~s}, 6 \mathrm{H}, \mathrm{CH}_{3}\right), 1.50(\mathrm{~s}, 12 \mathrm{H}$, $\left.\mathrm{CH}_{3}\right), 1.60\left(\mathrm{~s}, 12 \mathrm{H}, \mathrm{CH}_{3}\right) .-{ }^{13} \mathrm{C}-\mathrm{NMR}\left(\mathrm{C}_{6} \mathrm{D}_{6}\right): \delta=11.47\left(\mathrm{CH}_{3}\right)$, $11.54\left(\mathrm{CH}_{3}\right), 27.11\left(\mathrm{CH}_{3}\right), 77.04\left(\mathrm{C}-\mathrm{CH}_{3}\right), 137.17\left(\mathrm{C}-\mathrm{CH}_{3}\right), 137.99$ $\left(\mathrm{C}-\mathrm{CH}_{3}\right), 262.27$ (CS). - MS, m/z (\%): $\left[\mathrm{M}^{+}\right] 314(25),\left[\mathrm{M}^{+}-\right.$ $\mathrm{Me}] 299(1),\left[\mathrm{M}^{+}-\mathrm{S}-\mathrm{H}\right] 281(3),\left[\mathrm{M}^{+}-\mathrm{Me}_{5} \mathrm{C}_{5}\right] 179(100)$, $\left[\mathrm{M}^{+}-\mathrm{Me}_{5} \mathrm{C}_{5}-\mathrm{Me}\right] 164(8),\left[\mathrm{Me}_{5} \mathrm{C}_{5}^{+}\right] 135$ (16).

\section{$\mathrm{C}_{21} \mathrm{H}_{30} \mathrm{~S}$ (314.5) Ber. C 80.19 H 9.61 Gef. C 79.81 H 9.63}

Kristallstrukturanalysen von 2, 5 und 6: Die Atomkoordinaten sind in Tab. 2-4 zusammengestellt.

Tab. 4. Atomkoordinaten $\left(\cdot 10^{4}\right)$ und äquivalente isotrope Schwingungsparameter von 6 (mit Standardabweichungen)

\begin{tabular}{|c|c|c|c|c|c|c|c|}
\hline Atom & $x$ & & $y$ & & $z$ & & $\mathrm{U}(\mathrm{eq})$ \\
\hline$S(1)$ & 0 & & 8441 & (1) & 2500 & & 60 (1) \\
\hline$C(1)$ & 0 & & 7368 & (3) & 2500 & & 31 (2) \\
\hline$C(2)$ & 166 & (4) & 6877 & (2) & 1637 & (2) & 27 (1) \\
\hline$c(3)$ & 1026 & (4) & 5978 & (2) & 1678 & (2) & 32 (1) \\
\hline$C(4)$ & -36 & (5) & 5370 & (2) & 1354 & (2) & 34 (1) \\
\hline$C(5)$ & -1638 & (4) & 5771 & (2) & 1094 & (2) & 32 (1) \\
\hline$c(6)$ & -1585 & (4) & 6638 & (2) & 1267 & (2) & 29 (1) \\
\hline$c(7)$ & 1083 & (5) & 7432 & (2) & 946 & $(2)$ & 48 (1) \\
\hline$c(8)$ & 2874 & (5) & 5867 & (3) & 1901 & (2) & 55 (2) \\
\hline$C(9)$ & 322 & (5) & 4398 & (2) & 1238 & (2) & 56 (2) \\
\hline$C(10)$ & -3078 & (5) & 5252 & (2) & 689 & (2) & $54 \quad(2)$ \\
\hline$(11)$ & -2942 & (5) & 7306 & (2) & 1131 & (2) & 47 (1) \\
\hline
\end{tabular}

$1,2,4,5,6,7,8,9,10,11$-Decamethyltetracyclo[5.3.1. $\left.0^{2,8} .0^{4,11}\right]$ undeca5,9-dien-3-on (7): Eine Lösung von $0.50 \mathrm{~g}$ (1.68 mmol) des Ketons 5 in $20 \mathrm{ml}$ Benzol wird $24 \mathrm{~h}$ unter Rückfluß erhitzt. Man entfernt das Solvens i.Vak. und erhält 7 als farbloses Pulver. Ausb. $0.50 \mathrm{~g}$ $(100 \%)$, Schmp. $134^{\circ} \mathrm{C} .-{ }^{1} \mathrm{H}-\mathrm{NMR}\left(\mathrm{C}_{6} \mathrm{D}_{6}\right): \delta=0.60$ (s, 3H, $\left.\mathrm{CH}_{3}\right)$, $0.74\left(\mathrm{~s}, 6 \mathrm{H}, \mathrm{CH}_{3}\right), 0.78,0.92,1.11\left(3 \mathrm{~s}, 3 \times 3 \mathrm{H}, 3 \mathrm{CH}_{3}\right), 1.34(\mathrm{q}$, $\left.{ }^{5} J_{\mathrm{HH}}=1.2 \mathrm{~Hz}, 3 \mathrm{H}, \mathrm{CH}_{3}\right), 1.40\left(\mathrm{q},{ }^{5} J_{\mathrm{HH}}=1.0 \mathrm{~Hz}, 3 \mathrm{H}, \mathrm{CH}_{3}\right), 1.41$ $\left(\mathrm{q},{ }^{5} J_{\mathrm{HH}}=1.2 \mathrm{~Hz}, 3 \mathrm{H}, \mathrm{CH}_{3}\right), 1.58\left(\mathrm{q},{ }^{5} J_{\mathrm{HH}}=1.0 \mathrm{~Hz}, 3 \mathrm{H}, \mathrm{CH}_{3}\right)$. ${ }^{13} \mathrm{C}$-NMR $\left(\mathrm{C}_{6} \mathrm{D}_{6}\right): \delta=8.88,9.71,10.31,10.78,11.61,12.74,13.75$, $15.92\left(\mathrm{CH}_{3}\right), 61.43,62.74,63.53,71.95,78.72,132.84,133.66,137.37$, $138.66\left(C-\mathrm{CH}_{3}\right), 210.70(\mathrm{CO}) .-\mathrm{MS}, m / z(\%):\left[\mathrm{M}^{+}\right] 298(5),\left[\mathrm{M}^{+}\right.$ $\left.-\mathrm{Me}_{5} \mathrm{C}_{5}\right] 163(2),\left[\mathrm{Me}_{5} \mathrm{C}_{5}^{+}\right] 135(100)$.

\section{$\mathrm{C}_{21} \mathrm{H}_{30} \mathrm{O}(298.5)$ Ber. C $84.51 \mathrm{H} 10.13$ Gef. C 84.59 H 10.08}

1,2,4,5,6,7,8,9,10,11-Decamethyltetracyclo[5.3.1. $\left.0^{2,8} \cdot 0^{4,11}\right]$-undeca5,9-dien-3-thion (8): Eine Lösung von $0.50 \mathrm{~g}(1.59 \mathrm{mmol})$ des Thioketons 6 in $20 \mathrm{ml}$ Benzol wird 90 min unter Rückfluß erhitzt. Nach Entfernen des Solvens i.Vak. erhält man 8 als hellrotes Pulver. Ausb. $0.50 \mathrm{~g} \mathrm{(100 \% ),} \mathrm{Schmp.} 160^{\circ} \mathrm{C} .-{ }^{1} \mathrm{H}-\mathrm{NMR}\left(\mathrm{C}_{6} \mathrm{D}_{6}\right): \delta=0.62$, $0.72,0.76,0.79,1.15,1.30\left(6 \mathrm{~s}, 6 \times 3 \mathrm{H}, 6 \mathrm{CH}_{3}\right), 1.34\left(\mathrm{q},{ }^{5} J_{\mathrm{HH}}=\right.$ $\left.1.2 \mathrm{~Hz}, 3 \mathrm{H}, \mathrm{CH}_{3}\right), 1.36,1.42\left(2 \mathrm{q},{ }^{5} J_{\mathrm{HH}}=0.9 \mathrm{~Hz}, 2 \times 3 \mathrm{H}, 2 \mathrm{CH}_{3}\right)$, $1.63\left(\mathrm{q},{ }^{5} J_{\mathrm{HH}}=1.2 \mathrm{~Hz}, 3 \mathrm{H}\right) .-{ }^{13} \mathrm{C}-\mathrm{NMR}\left(\mathrm{C}_{6} \mathrm{D}_{6}\right): \delta=9.83,10.04$, $10.40,11.41,11.55,12.80,13.11,13.95,15.76,18.56\left(\mathrm{CH}_{3}\right), 62.38$, $63.32,64.00,72.43,75.75,88.22,133.41,135.68,138.15,139.19$ $\left(C-\mathrm{CH}_{3}\right), 264.96(\mathrm{CS}) .-\mathrm{MS}, m / z(\%):\left[\mathrm{M}^{+}\right] 314(19),\left[\mathrm{M}^{+}-\right.$
SH] 281 (2), $\left[\mathrm{M}^{+}-\mathrm{Me}_{5} \mathrm{C}_{5}\right] 179(100),\left[\mathrm{M}^{+}-\mathrm{Me}_{5} \mathrm{C}_{5}-\mathrm{CH}_{3}\right]$ $164(2),\left[\mathrm{Me}_{5} \mathrm{C}_{5}^{+}\right] 135(16)$.

\section{$\mathrm{C}_{21} \mathrm{H}_{30} \mathrm{~S}$ (314.5) Ber. C 80.19 H 9.61 Gef. C 79.72 H 9.85}

Photochemisch induzierte $[4+2]$-Cycloaddition des Thioketons $6 z u$ 8: Eine Lösung von $0.20 \mathrm{~g}(0.64 \mathrm{mmol}) 6$ in $150 \mathrm{ml}$ Pentan wird in einem Photoreaktor mit einer 150-W-Hg-Hochdrucklampe 2 min bestrahlt. Man erhält $\mathbf{8}$ als hellrotes Pulver. Ausb. quantitativ. - Analytische Daten wie oben.

${ }^{[1]}$ L. de Vries, J. Org. Chem. 1960, 25, 1838.

${ }^{[2]}$ R. J. Bushby, D. W. Jones, J. Chem. Soc., Chem. Commun. 1979, $688-690$.

${ }^{[3]}$ U. Burger, A. Delay, F. Mazenod, Helv. Chim. Acta 1974, 57, $2106-2111$.

${ }^{[4]}$ R. F. Childs, M. Zeya, J. Am. Chem. Soc. 1974, 96, 6418-6424.

${ }^{\left[{ }^{5}\right]}$ H.-A. Brune, P. Lach, G. Schmidtberg, Chem. Ber. 1985, 118, $2671-2680$.

${ }^{[6]}$ H.-A. Brune, P. Lach, G. Schmidtberg, Chem. Ber. 1985, 118, $2681-2691$.

${ }^{[7]}$ F. X. Kohl, P. Jutzi, Chem. Ber. 1987, 120, 1539-1543.

${ }^{[8]}$ P. Jutzi, D. Kanne, C. Krüger, Angew. Chem. 1986, 98, 163-164.

[9] P. Jutzi, F. X. Kohl, P. Hofmann, C. Krüger, Y.-H. Tsay, Chem. Ber. 1980, 113, 757-769.

${ }^{[10]}$ J. L. Atwood, W. E. Hunter, J. Chem. Soc., Chem. Commun. $1981,925-927$.

[i1] P. Jutzi, A. Mix, in Vorbereitung.

${ }^{[12]}$ Eine Diels-Alder-Reaktion zwischen zwei $\mathrm{Me}_{5} \mathrm{C}_{5}$-Fragmenten ist in den bisher bekannten mono- und bis- $\eta^{1}-\mathrm{Cp}^{*}$-Verbindungen nicht beobachtet worden.

${ }^{[13]}$ Bisher wurden die Festkörperstrukturen folgender $\mathrm{Cp}_{2}^{*}-\mathrm{Haupt}-$ gruppenelement-Verbindungen bekannt: $\left(\mathrm{Me}_{5} \mathrm{C}_{5}\right)_{2} \mathrm{BF}$ : P. Jutzi, B. Krato, M. Hursthouse, Chem. Ber. 1987, 120, 565-574; $\left(\mathrm{Me}_{5} \mathrm{C}_{5}\right)_{2} \mathrm{SiX}_{2}(\mathrm{X}=\mathrm{F}, \mathrm{Cl})$ : P. Jutzi, D. Kanne, M. Hursthouse, A. J. Howes, ibid. 1988, 121, $1299-1305 ;\left(\mathrm{Me}_{5} \mathrm{C}_{5}\right)_{2} \mathrm{~S}:$ A. J. Bard, A. H. Cowley, J. K. Leland, G. J. N. Thomas, N. C. Norman, P. Jutzi, C. P. Morley, E. Schlüter, J. Chem. Soc., Dalton Trans. 1985, 1303-1307.

${ }^{[14]}$ K. N. Raymond, C. W. Eigenbrot, Acc. Chem. Res. 1980, 13, $276-283$.

[15] Diels-Alder-Reaktionen sind sowohl unter präparativen als auch mechanistischen Aspekten ausführlich diskutiert worden. Intramolekulare Diels-Alder-Reaktionen: G. Brieger, J. N. Bennett, Chem. Rev. 1980, 80, 63-97. Zum Mechanismus der DielsAlder-Reaktion: J. Sauer, R. Sustmann, Angew. Chem. 1980, 92, 773-801; Angew. Chem. Int. Ed. Engl. 1980, 19, 779.

${ }^{[16]}$ Die Absorptionskorrektur wurde mit dem Programm XABS durchgeführt: H. Hope, B. Moezzi, University of California, Davis. Dieses Programm erhält einen Absorptionstensor aus den $F_{0}-F_{\mathrm{c}}$-Werten. B. Moezzi, Ph. D. Dissertation, University of California, Davis, 1987.

[17] Weitere Einzelheiten zu den Kristallstrukturanalysen von 2, 5 und 6 können beim Fachinformationszentrum Karlsruhe, Gesellschaft für wissenschaftlich-technische Information $\mathrm{mbH}$, D-7514 Eggenstein-Leopoldshafen 2, unter Angabe der Hinterlegungsnummer CSD-56702, der Autorennamen und des Zeitschriftenzitats angefordert werden.

[260/92]

\section{CAS-Registry-Nummern}

1: 51905-34-1 / 2: 108561-47-3 / 3: 143171-48-6 / 4: 26167-94-2/ 5: 143171-49-7 / 6: 143171-50-0/7: 143171-51-1 / 8: 143171-52-2/ Pentamethylcyclopentadien: 41539-64-4 